\title{
THE IDEAL CLASS GROUPS OF TWO CYCLOTOMIC FIELDS
}

\author{
FRANK GERTH III
}

\begin{abstract}
ABSTRACr. It is known that there are exactly two cyclotomic fields with class numbers equal to 8 , namely the fields of 29 th and 68 th roots of unity. We show by elementary methods how to determine the structure of the ideal class groups of these fields.
\end{abstract}

In [2] Masley indicates that there are exactly two cyclotomic fields that have class numbers equal to 8 . These fields are $\mathbf{Q}\left(\zeta_{29}\right)$ and $\mathbf{Q}\left(\zeta_{68}\right)$, where $\mathbf{Q}$ denotes the field of rational numbers, and $\zeta_{29}$ (resp., $\zeta_{68}$ ) is a primitive 29th (resp., 68th) root of unity. Masley remarks that it is known that the ideal class group of $\mathbf{Q}\left(\zeta_{29}\right)$ has exponent 2 but that the structure of the ideal class group of $\mathbf{Q}\left(\zeta_{68}\right)$ is unknown. In this paper we show by elementary methods how to determine the structure of the ideal class groups of $\mathbf{Q}\left(\zeta_{29}\right)$ and $\mathbf{Q}\left(\zeta_{68}\right)$, given that their class numbers are equal to 8.

We let $K$ be a finite extension of $Q$, and we let $L$ be an extension of $K$ of degree 2. We let $\sigma$ be the generator of $\operatorname{Gal}(L / K)$. We let $C_{K}$ (resp., $C_{L}$ ) denote the ideal class group of $K$ (resp., $L$ ) and $h_{K}$ (resp., $h_{L}$ ) the class number of $K$ (resp., $L$ ). We shall suppose that $h_{K}=1$.

LEMMA 1. If $a \in C_{L}$, then $a^{1+\sigma}=1$.

Proof. Let $N: C_{L} \rightarrow C_{K}$ be the map induced by the norm map from ideals of $L$ to ideals of $K$, and let $i: C_{K} \rightarrow C_{L}$ be the map induced by the inclusion map from ideals of $K$ to ideals of $L$. Then $a^{1+\sigma}=i(N a)=1$ since $N a \in C_{K}$ and $h_{K}=1$.

Next we let $B_{L}=\left\{a \in C_{L} \mid a^{2}=1\right\}$ and $A_{L}=\left\{a \in C_{L} \mid a^{\sigma}=a\right\}$. If $a \in A_{L}$, then $a^{2}=a^{1+\sigma}=1$ by Lemma 1 , and hence $A_{L} \subseteq B_{L}$. On the other hand, if $a \in B_{L}$, then $a^{2}=1$ implies $a=a^{-1}$. From Lemma $1, a^{\sigma}=a^{-1}$. So $a^{\sigma}=a$, and hence $a \in A_{L}$. So we have proved the following lemma.

LemMa 2. $B_{L}=A_{L}$.

LEMMA 3. $\left|A_{L}\right|=2^{d-1-(r+1-q)}$ where $\left|A_{L}\right|$ denotes the order of $A_{L}$, $d$ is the number of ramified primes in $L / K, q$ satisfies $2^{q}=\left|\left(E_{K} \cap N_{L / K} L^{*}\right) / E_{K}^{2}\right|$ with $E_{K}$ the group of units of $K$ and $N_{L / K}$ the norm map from $L$ to $K$, and $r$ is the rank of the free abelian part of $E_{K}$.

Proof. This result is a classical result proved in [1, Theorem 13].

REMARK. $r+1-q \geqslant 0$ in Lemma 3.

Received by the editors February 15, 1979 and, in revised form, April 24, 1979.

AMS (MOS) subject classifications (1970). Primary 12A35, 12 A50.

(C) 1980 American Mathematical Society 0002-9939/80/0000-0106/\$01.50 
Now we consider $K=\mathbf{Q}\left(\zeta_{17}\right)$ and $L=\mathbf{Q}\left(\zeta_{68}\right)=K(\sqrt{-1})$. It is well known that $h_{K}=1$. We shall apply Lemma 3 to $L=\mathbf{Q}\left(\zeta_{68}\right)$. It is easy to see that the only primes of $K$ which ramify in $L$ are the primes of $K$ that are above the rational prime 2. A simple calculation shows that the order of 2 modulo 17 is 8 . Hence 2 decomposes in $\mathbf{Q}(\sqrt{17})$, but the primes above 2 in $\mathbf{Q}(\sqrt{17})$ remain inert in $\mathbf{Q}\left(\zeta_{17}\right)$. So there are exactly 2 primes of $K$ which ramify in $L$. So $d=2$ in Lemma 3, and we see that $\left|A_{L}\right| \leqslant 2$. Since $h_{L}=8$ by [2], then $\left|B_{L}\right|>1$. So $\left|B_{L}\right|=\left|A_{L}\right|=2$, and hence $C_{L}$ is a cyclic group of order 8 . So we have proved the second part of the following theorem.

THEOREM 1. The ideal class group of $\mathbf{Q}\left(\zeta_{29}\right)$ is an elementary abelian 2-group of order 8, and the ideal class group of $\mathrm{Q}\left(\zeta_{68}\right)$ is a cyclic group of order 8.

REMARK. Although the first part of Theorem 1 was already known, we briefly indicate how to prove that result. We let $K=\mathbf{Q}(\sqrt{29})$, and we let $L$ be the subfield of $\mathbf{Q}\left(\zeta_{29}\right)$ of degree 2 over $K$. In applying Lemma 3, calculations show that $d=3$ (the prime above 29 and the two real archimedean primes of $K$ ramify in $L / K), r=1$, and $q=0$. So $\left|A_{L}\right|=1$, and hence $\left|B_{L}\right|=1$. Next we let $M=Q\left(\zeta_{29}\right)$, and we let $C_{M}$ denote the ideal class group of $M$. We define $B_{M}=\left\{a \in C_{M} \mid a^{2}=\right.$ $1\}$, and $A_{M}=\left\{a \in B_{M} \mid a^{\tau}=a\right\}$. Here $\tau$ is a generator of $\operatorname{Gal}(M / L)$, which is a cyclic group of order 7. Now we note that $\left|A_{M}\right|=1$. (If $a \in A_{M}$, then $a=a^{7}=$ $a^{1+\tau+\tau^{2}+\cdots+\tau^{6}}=1$.) But $\left|B_{M}\right| \neq 1$ since $\left|C_{M}\right|=8$. Hence $\tau$ is a automorphism of $B_{M}$ of degree 7, which forces $\left|B_{M}\right|=\left|C_{M}\right|=8$.

REMARK. For certain facts about cyclotomic fields that are used in this paper, see [3].

\section{REFERENCES}

1. H. Hasse, Bericht über neuere Untersuchungen und Probleme aus der Theorie der algebraischen Zahlkörper. Ia, Jber. Deutsch. Math.-Verein. 36 (1927), 233-311.

2. J. Masley, Solution of small class number problems for cyclotomic fields, Compositio Math. 33 (1976), 179-186.

3. E. Weiss, Algebraic number theory, McGraw-Hill, New York, 1963.

Department of Mathematics, University of TeXas, Austin, TeXas 78712 